grupo etário dos 61 aos 80 anos, relacionado com traumatismos de baixa energia. ${ }^{7}$ Os fatores de risco epide-

\title{
Luxação do Ombro: Avaliação e Tratamento
}

Dr. Jóni Nunes, Dr. André Sarmento, Prof. Dra. Cristina Valente, Dr. Renato Andrade, Prof. Dr. João Espregueira-Mendes

Clínica do Dragão, Espregueira-Mendes Sports Centre - FIFA Medical Centre of Excellence, Porto.

\section{RESUMO / ABSTRACT}

A luxação anterior do ombro, sendo a luxação mais frequente do corpo humano, é uma condição que afeta os atletas profissionais e amadores, particularmente em desportos overhead ou de contacto. Apesar da sua elevada incidência, várias questões permanecem no seio do debate científico, com muitas questões ainda por esclarecer, tais como: qual o tipo e a duração de imobilização; qual o melhor tratamento para cada tipo de atleta; qual a melhor opção para o atleta que sofre o primeiro episódio de luxação do ombro durante a época desportiva; quais os critérios de retorno ao desporto. Este artigo pretende expor o estado da arte atual acerca desta temática.

Anterior shoulder dislocation, as the most common dislocation in humans, is a condition affecting both professional and recreational athletes, particularly in overhead and contact sports. Despite its high incidence, several questions remain within the scientific debate, with many questions still to be clarified, such as: which type and duration of immobilization; what is the best treatment for each type of athlete; what is the best option for the athlete who suffers the first episode of shoulder dislocation during the sports season; which are the return to sports criteria. This article intends to expose the current state of the art on this theme.

\section{PALAVRAS-CHAVE / KEYWORDS}

Luxação do ombro, luxação anterior, lesão Bankart, lesão Hill-Sachs, tratamento Shoulder dislocation, anterior dislocation, Bankart injury, Hill-Sachs injury, treatment

\section{Introdução}

O ombro é a articulação do corpo com maior arco de mobilidade, graças ao seu desenho anatómico. ${ }^{1}$ Esta particularidade confere, no entanto, uma maior predisposição para a instabilidade, já que em qualquer momento do arco de movimento apenas 25\% da superfície articular da cabeça umeral contacta com a superfície da glenoide, dependendo em larga medida da complexa interação entre os estabilizadores estáticos (estruturas capsuloligamentares, ósseas e labrais) e dinâmicos (componente neuromuscular). ${ }^{2}$

\section{Tipos de instabilidade}

A instabilidade do ombro pode ser dividida em dois grandes grupos: instabilidade atraumática e traumática. Instabilidade atraumática está relacionada com certas condições intrínsecas (por exemplo, doença de Ehlers-Danlos, síndrome de Marfan) ou extrínsecas (microtraumas resultantes do overuse em atletas) que predispõem o indivíduo a eventos de (sub)luxações do ombro. ${ }^{3}$ A instabilidade traumática é resultante de um traumatismo direto ou indireto do ombro e é classificada segundo a direção da cabeça umeral em relação à superfície glenoideia, resultando em luxações anteriores, posteriores ou inferiores (luxatio erecta). ${ }^{4}$ A luxação anterior do ombro representa cerca de $98 \%$ das luxações traumáticas, sendo a luxação mais frequente do corpo humano, pelo que iremos abordar mais detalhadamente.

\section{Luxação traumática anterior do ombro}

\section{Epidemiologia}

A incidência na população é de cerca de 23.9/100.00 habitantes, com uma taxa de incidência anual de 1,7\% na população em geral, mais frequente no sexo masculino. ${ }^{5}$ Apresenta uma distribuição bimodal, com um pico de incidência no grupo etário dos 15 aos 29 anos, relacionado com a prática desportiva e mecanismos traumáticos de alta cinética ${ }^{6}$ e outro pico de incidência mais baixo no miológicos incluem o sexo masculino, a idade inferior a 30 anos e a prática de desportos de contacto, sendo o futebol, o hóquei e o wrestling as modalidades mais com maior risco. ${ }^{8}$

\section{Avaliação clínica}

A história e avaliação clínica são fundamentais para o diagnóstico e podem conduzir a um elevado grau de suspeição de laxição anterior. 0 mecanismo lesional é, regra geral, um traumatismo direto com o ombro numa posição de abdução e rotação externa, provocando um quadro de impotência funcional súbita. Ao exame objetivo, o paciente apresenta-se numa posição antálgica com o ombro em rotação interna em ligeira abdução e o cotovelo a $90^{\circ}$ de flexão (figura 1), devendo o clínico procurar sinais de luxação anterior, como sejam: (1) sinal da cruzeta (incapacidade de palpação da cabeça umeral na face lateral do ombro); (2) perda do normal contorno do ombro com uma exacerbada proeminência acromial em comparação com o lado contralateral, havendo a possibilidade de palpação direta da cabeça umeral numa posição anterior em indivíduos com biótipo ectomorfo.

\section{Emergência clínica}

A redução da luxação do ombro constitui uma emergência clínica. Porém, pelas possíveis consequências neurovasculares e articulares, a manobra de recolocação nunca deverá ser realizada sem que antes se realize uma radiografia da articulação, com dupla incidência, de forma a excluir condições associadas, como seja a fratura-luxação do

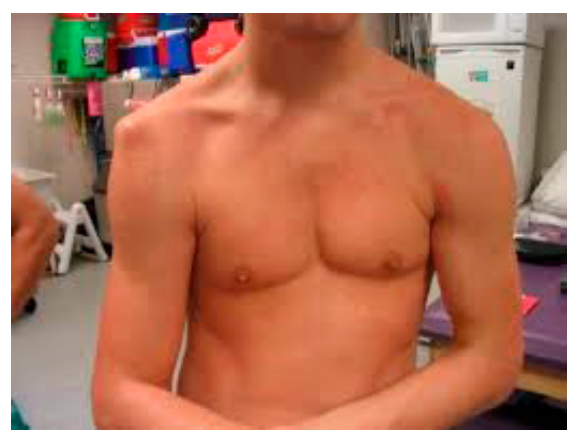

Figura 1 - Luxação anterior do ombro direito (https://ombroecotovelodf.com.br/ instabilidade-do-ombro/) 
ombro, que deve orientar as diretrizes do tratamento. A exceção a esta regra aplica-se quando, por motivos logísticos, o acesso à radiografia não esteja disponível em tempo útil.

Uma vez que o sucesso da redução seja confirmado radiograficamente, deve ser realizada uma avaliação neurovascular do membro que fará parte do algoritmo clínico. A avaliação neurovascular, conhecendo a tríade patognomónica desta lesão (luxação do ombro, diminuição ou ausência de pulsos periféricos e a presença de um tumefacto hematoma axilar), é importante, pois existe uma taxa de cerca de 10\% de neuropraxia do nervo axilar ${ }^{9}$ e de cerca de 1-2\% de lesão da artéria axilar. ${ }^{10}$

\section{Imobilização}

A imobilização da articulação do ombro após um evento de luxação anterior permanece em debate científico. A maioria dos indivíduos são tratados com imobilização do ombro em rotação interna, com recurso a ortótese, durante 2 a 3 semanas após o evento inicial. ${ }^{11}$ Este tipo de imobilização foi questionado pela primeira vez em 199912, uma vez que imobilização em rotação externa era superior, já que permitia melhor contacto da lesão labral com a superfície glenoideia que, em teoria, conduziria a melhor taxa de cicatrização. No entanto, estudos mais recentes têm falhado em demonstrar a superioridade de um tipo de imobilização sobre a outra em relação à diminuição da taxa de recorrência e melhoria da qualidade de vida dos pacientes. ${ }^{13}$

Em relação à duração, a imobilização por um período superior a uma semana não demonstrou superioridade na taxa de recorrência em relação à imobilização por períodos mais curtos. Neste contexto a individualização do tratamento tendo em conta o controlo álgico parece ser a melhor estratégia. ${ }^{14}$

\section{Lesões associadas}

As lesões associadas à luxação anterior do ombro podem dividir-se em lesões labrais/cartilagíneas (ex: lesão de Bankart), lesões ósseas (ex: lesão de Hill-Sachs), lesões da coifa dos rotadores e lesões neurovasculares:

- Lesão de Bankart: É uma avulsão do labrum anterior e do feixe anterior do ligamento glenoumeral inferior da sua inserção na face ântero-inferior da glenoide. Esta lesão pode constituir um defeito puramente capsulo-ligamentar (Soft Bankart), com uma prevalência estimada de cerca de 73\%, ou associado a um componente ósseo (Bony Bankart), com uma prevalência estimada de cerca de $49 \%$, geralmente em contexto de luxações recorrentes ${ }^{15}$

- Lesão de Hill-Sachs: É uma depressão cortical na face póstero-lateral da cabeça do úmero por impactação na vertente ântero-inferior do rebordo da glenoide. Tem uma prevalência estimada de 54\%. ${ }^{16}$

\section{Decisão terapêutica}

A orientação terapêutica deve ser adaptada ao indivíduo e ao seu contexto. Assim, há questões que devem ser respondidas antes da decisão terapêutica, tais como: Qual o sexo e a idade? Foi o primeiro episódio? É atleta amador ou profissional? Tem lesões associadas? Tem hiperlaxidão ligamentar?

Após respondidas as questões elementares da recolha cuidada da história clínica e exame clínico minucioso, há outros fatores que devem ser tidos em conta:

1. A recorrência da instabilidade do ombro é diretamente proporcional ao nível de atividade e inversamente proporcional à idade no primeiro episódio ${ }^{17}$

2. A taxa de recorrência é de cerca de $72 \%$ quando o primeiro episódio acontece antes dos 23 anos de idade e de cerca de $27 \%$ após os 30 $\operatorname{anos}^{18}$

3. Alterações degenerativas da articulação glenoumeral 25 anos após o primeiro episódio devem ser esperadas num grau moderado em cerca de $27 \%$ dos casos, e num grau moderado a severo em cerca de $34 \%$ dos casos. ${ }^{18}$

\section{Algoritmo terapêutico em atletas}

Na primeira etapa da decisão terapêutica de um atleta com instabilidade anterior do ombro deve fazer-se a exclusão de hiperlaxidão ligamentar e discinesia escapulotorácica, as quais devem alterar a conduta terapêutica.

As indicações para o tratamento não-cirúrgico definem-se como um atleta-tipo ideal, atleta com uma lesão in-season, praticante de desporto de não-contacto ou overhead e que responda favoravelmente ao tratamento não-cirúrgico, sem apreensão ou instabilidade evidente. $\mathrm{O}$ tratamento não-cirúrgico deve focar o ganho progressivo de mobilidade, força, propriocepção e, acima de tudo, autoconfiança. Por outro lado, o tratamento cirúrgico será o gold-standard num atleta com uma lesão off-season, com menos de 20 anos de idade e praticante de desporto de contacto ou overhead. ${ }^{14}$

\section{A influência do conceito on-track e off-track na decisão cirúrgica}

A compreensão da complexa biomecânica da articulação do ombro exige uma avaliação dinâmica da interação anatómica tridimensional entre a superfície articular da cabeça umeral com a superfície glenoideia, sendo que esta interação é dramaticamente alterada no contexto de episódios de luxação.

Sabe-se hoje que uma perda de cerca de $30 \%$ da superfície da glenoide leva a um aumento das

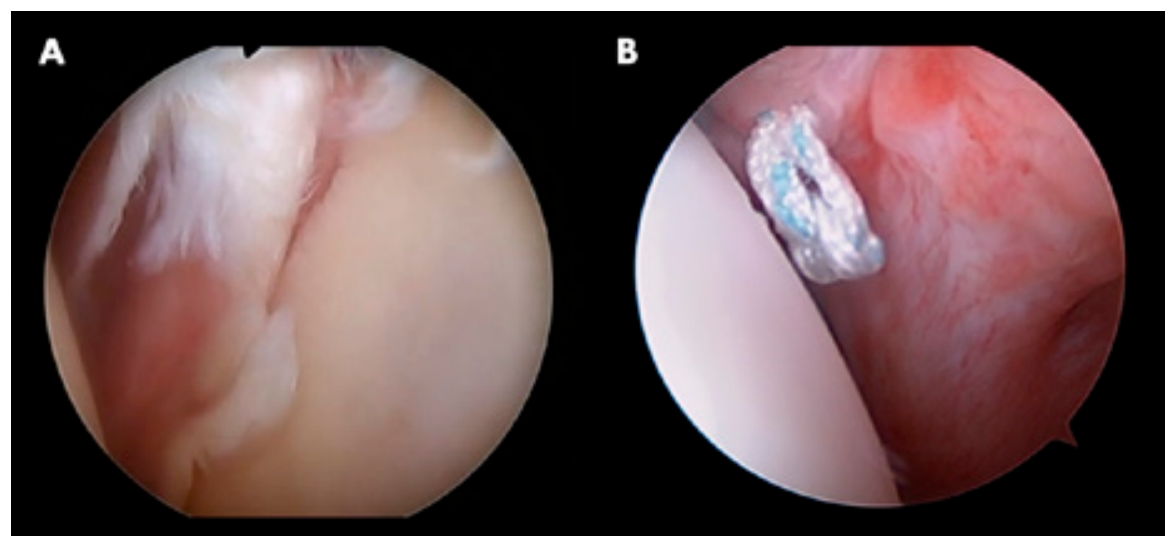

Figura 2 - A) Lesão tipo Soft-Bankart; B) Reparação artroscópica de lesão de Bankart com âncoras tecidulares 
pressões de contacto em cerca de 300 a 400\% no bordo ântero-inferior da superfície da glenoide ${ }^{19}$, facto que parece justificar a falência de abordagens cirúrgicas mais conservadoras neste contexto.

No ano 2000, Burkhart et al. ${ }^{20}$ definiram os conceitos de lesões tipo engaging e non-engaging, tendo sido complementado em 2014 por Giacomo et al., ${ }^{21}$ que propõem um conceito de instabilidade bipolar, definindo

- Lesões on-track aquelas em que o defeito de Hill-Sachs está contido pelo diâmetro do curso articular da glenoide em todo o arco de movimento, sendo por isso lesões do tipo non-engaging

- Lesões off-track as do tipo engaging, já que em algum momento do arco de movimento o defeito de Hill-Sachs não está contido pela superfície articular da glenoide. Assim, o algoritmo cirúrgico mais aceite atualmente tem em conta este tipo de alterações:

1. Indivíduos com defeito ósseo da glenoide inferior a 25\%:

a) e/ou lesão de Hill-Sachs on-track: operação de Bankart artroscópica (reinserção labral na sua posição anatómica) (figura 2);

b) e lesão de Hill-Sachs off-track: operação de Bankart artroscópica combinada com remplissage (capsulotenodese do tendão do músculo infraespinhoso ao defeito ósseo).

2. Indivíduos com defeito ósseo da glenoide superior a $25 \%$ :

a.) e lesão de Hill-Sachs on-track: procedimento de Bristow-Latarget (transferência da apófise coracoide para o bordo ântero-inferior da glenoide);

b) e lesão de Hill-sachs off-track: procedimento de Bristow-Latarget combinado com procedimento de remplissage ou preenchimento do defeito umeral com enxerto ósseo.

\section{Retorno ao desporto}

A escassez de publicações na literatura acerca desta temática dificulta a gestão de expectativas dos atletas. Apesar das inúmeras variações entre protocolos, as diferentes fases apresentam pilares comuns com critérios bem definidos para a progressão entre as fases de reabilitação:

1. Fase 1 (cerca de 4 semanas): imobilização, exercícios pendulares, contrações isométricas;
2. Fase 2 (cerca de 4 semanas): trabalho progressivo de mobilidade passiva, início de trabalho de mobilidade ativa sem resistência (limite de $45^{\circ}$ de rotação externa);

3. Fase 3 (cerca de 4 semanas): trabalho de mobilidade ativa com resistência (limite de $90^{\circ}$ de rotação externa);

4. Fase 4 (pelo menos 4 semanas): pliometria, feedback propriocetivo e exercícios específicos da modalidade.

Após o tratamento não-cirúrgico, e apesar de não existirem, até ao momento, guidelines que orientem o return-to-play, é globalmente aceite que o atleta estará apto quando possuir arco de mobilidade e força simétricas ao membro contralateral e conclua exercícios específicos da modalidade sem dor. No entanto, a taxa de instabilidade recorrente é ainda muito elevada, diminuindo a probabilidade de sucesso de retorno ao desporto. ${ }^{22}$ Por exemplo, Buss et al. reportaram que 27 em 30 atletas conseguiram voltar à sua atividade desportiva ao nível pré-lesional numa média de 10,2 dias após o tratamento não-cirúrgico, mas que 37\% destes atletas apresentavam instabilidade recorrente. ${ }^{23}$ Também Dickens et al. reportaram, num estudo prospetivo, uma taxa de $73 \%$ de retorno ao desporto um episódio de instabilidade, numa média de cinco dias após a lesão. Destes, 36\% não reportaram outro episódio de instabilidade, 30\% sofreram pelo menos uma recidiva, mas conseguiram completar a época em curso e $33 \%$ sofreram uma nova luxação e não completaram a época desportiva. ${ }^{24}$

Os atletas com instabilidade recorrente e que são incapazes de realizar os exercícios específicos do desporto são indicados para estabilização cirúrgica. Num estudo comparativo, dos atletas que realizaram estabilização artroscópica, 90\% voltaram a competir na temporada seguinte sem recorrência de instabilidade. Estes tinham cerca de seis vezes mais probabilidade de retomarem à competição sem instabilidade recorrente na temporada seguinte em comparação com atletas que foram submetidos a tratamento não-cirúrgico. ${ }^{25}$ Dentro das abordagens cirúrgicas, a estabilização artroscópica tem-se tornado mais populares e com altas taxas de sucesso. ${ }^{26}$ Numa revisão sistemática, com 1036 pacientes submetidos a cirurgia de estabilização do ombro, o retorno ao desporto foi mais elevado após reparo de Bankart artroscópico (71\%) ou o procedimento de Latarjet (73\%) do que a estabilização aberta (66\%). ${ }^{27}$ Existe depois uma miríade de protocolos de reabilitação pós-cirúrgica que visam otimizar o tempo de retorno à competição, sendo que o tempo médio varia entre 4 a 6 meses $^{28}$ e deve ser ponderado quando presentes valores objetivos de força durante a abdução e rotação externa simétricas ao membro contralateral.

Os autores declaram ausência de conflito de interesses, assim como a originalidade do texto e a sua não publicação prévia.

Correspondência: joninunesortopedia@ gmail.com

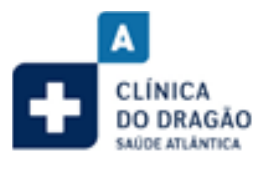

\section{Bibliografia}

1. Arliani GG, Astur DdC, Cohen C, et al. Surgical versus nonsurgical treatment in first traumatic anterior dislocation of the shoulder in athletes. Open Access J Sports Med. 2011; 2:19-24.

2. Mascarenhas R, Rusen J, Saltzman BM, et al. Management of humeral and glenoid bone loss in recurrent glenohumeral instability. Adv Orthop. 2014; 2014:640952-52.

3. Pope EJ, Ward JP, Rokito AS. Anterior shoulder instability - a history of arthroscopic treatment. Bull NYU Hosp Jt Dis. 2011; 69(1):44-9.

4. Zacchilli MA, Owens BD. Epidemiology of shoulder dislocations presenting to emergency departments in the United States. J Bone Joint Surg Am. 2010; 92(3):542-9.

5. Hayes K, Callanan M, Walton J, et al. Shoulder instability: management and rehabilitation. J Orthop Sports Phys Ther. 2002; 32(10):497509.

6. Rumian A, Coffey D, Fogerty S, et al. Acute first-time shoulder dislocation. Orthop Trauma. 2011; 25(5):363-68.

7. Cutts S, Prempeh M, Drew S. Anterior shoulder dislocation. Ann R Coll Surg Engl. 2009; 91(1):2-7.

8. Owens BD, Agel J, Mountcastle SB, et al. Incidence of glenohumeral instability in collegiate athletics. Am J Sports Med. 2009; 37(9):1750-4.

9. Visser CP, Coene LN, Brand R, et al. The incidence of nerve injury in anterior dislocation of the shoulder and its influence on functional recovery. A prospective clinical and EMG study. J Bone Joint Surg Br. 1999; 81(4):679-85. 
10. Stayner LR, Cummings J, Andersen J, et al. Shoulder dislocations in patients older than 40 years of age. Orthop Clin North Am. 2000; 31(2):231-9.

11. Jakobsen BW, Johannsen HV, Suder P, et al. Primary repair versus conservative treatment of first-time traumatic anterior dislocation of the shoulder: a randomized study with 10-year follow-up. Arthroscopy. 2007; 23(2):118-23.

12. Itoi E, Hatakeyama Y, Urayama M, et al. Position of immobilization after dislocation of the shoulder. A cadaveric study. J Bone Joint Surg Am. 1999; 81(3):385-90.

13. Gutkowska O, Martynkiewicz J, Gosk J. Position of Immobilization After First-Time Traumatic Anterior Glenohumeral Dislocation: A Literature Review. Med Sci Monit. 2017; 23:3437-45.

14. Owens BD, Dickens JF, Kilcoyne KG, et al. Management of mid-season traumatic anterior shoulder instability in athletes. J Am Acad Orthop Surg. 2012; 20(8):518-26.

15. Widjaja AB, Tran A, Bailey M, et al. Correlation between Bankart and Hill-Sachs lesions in anterior shoulder dislocation. ANZ J Surg. 2006; 76(6):436-8.

16. Hovelius L, Augustini BG, Fredin H, et al. Primary anterior dislocation of the shoulder in young patients. A ten-year prospective study. J Bone Joint Surg Am. 1996; 78(11):1677-84.

17. Schrumpf MA, Maak TG, Delos D, et al. The management of anterior glenohumeral instability with and without bone loss: AAOS exhibit selection. J Bone Joint Surg Am. 2014; 96(2):e12.

18. Hovelius L, Rahme H. Primary anterior dislocation of the shoulder: long-term prognosis at the age of 40 years or younger. Knee Surg Sports Traumatol Arthrosc. 2016; 24(2):33042.

19. Greis PE, Scuderi MG, Mohr A, et al. Glenohumeral articular contact areas and pressures following labral and osseous injury to the anteroinferior quadrant of the glenoid. J Shoulder Elbow Surg. 2002; 11(5):442-51.

20. Burkhart SS, De Beer JF. Traumatic glenohumeral bone defects and their relationship to failure of arthroscopic Bankart repairs: significance of the inverted-pear glenoid and the humeral engaging Hill-Sachs lesion. Arthroscopy. 2000; 16(7):677-94.

21. Di Giacomo G, Itoi E, Burkhart SS. Evolving concept of bipolar bone loss and the Hill-Sachs lesion: from "engaging/non-engaging" lesion to "on-track/off-track" lesion. Arthroscopy. 2014; 30(1):90-8.

22. Elsenbeck MJ, Dickens JF. Return to Sports After Shoulder Stabilization Surgery for Anterior Shoulder Instability. Curr Rev Musculoskelet Med. 2017; 10(4):491-98.

23. Buss DD, Lynch GP, Meyer CP, et al. Nonoperative management for in-season athletes with anterior shoulder instability. Am J Sports Med 2004;32(6):1430-3.

24. Dickens JF, Owens BD, Cameron KL, et al. Return to play and recurrent instability after in-season anterior shoulder instability: a prospective multicenter study. Am J Sports Med. 2014; 42(12):2842-50.

25. Dickens JF, Rue JP, Cameron KL, et al. Successful Return to Sport After Arthroscopic Shoulder Stabilization Versus Nonoperative Managemen in Contact Athletes With Anterior Shoulder Ins- tability: A Prospective Multicenter Study. Am J Sports Med. 2017; 45(11):2540-46.

26. Horner NS, Ekhtiari S, Chan AA, et al. Joint Specific Return to Play Recommendations: Return to Play in Non-operative Hip/Groin Pain. In: Musahl V, Karlsson J, Krutsch W, Mandelbaum BR, Espregueira-Mendes J, d'Hooghe P, eds. Return to Play in Football: An Evidence-based Approach. Springer Berlin Heidelberg. 2018; 331-46.

27. Ialenti MN, Mulvihill JD, Feinstein M, et al. Return to Play Following Shoulder Stabilization: A Systematic Review and Meta-analysis. Orthop Sports Med. 2017; 5(9):2325967117726055-55.

28. Pagnani MJ, Dome DC. Surgical treatment of traumatic anterior shoulder instability in american football players. J Bone Joint Surg Am. 2002; 84(5):711-5 